\title{
Evaluation of the Neuroprotective Potential of N-Acetylcysteine for Prevention and Treatment of Cognitive Aging and Dementia
}

\author{
Y. Hara, N. McKeehan, P.A. Dacks, H.M. Fillit \\ Alzheimer's Drug Discovery Foundation, 57 West 57th St. Suite 904, New York, NY 10019, USA
}

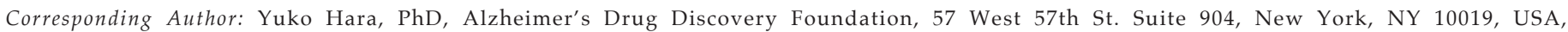
Email: yhara@alzdiscovery.org, Phone: 1-212-901-7991, Fax: 1-212-901-8010

J Prev Alz Dis 2017;4(3):201-206

Published online June 13, 2017, http:/ / dx.doi.org/10.14283/jpad.2017.22

\begin{abstract}
Alzheimer's disease is a progressive neurodegenerative disease for which there is no cure and only a few treatments providing little relief. Increased oxidative stress that is associated with aging is strongly implicated in the pathogenesis and progression of Alzheimer's disease. Studies have shown that levels of the endogenous antioxidant glutathione decline at an early stage of Alzheimer's disease with decreased levels correlating with worse cognitive functions. N-acetylcysteine, a drug also widely available as a dietary supplement, is a precursor of L-cysteine, which in turn is a component of glutathione. Because cysteine availability is a limiting factor for glutathione synthesis, treatment with $\mathrm{N}$-acetylcysteine may increase glutathione levels and thereby counter oxidative stress, promote redox -regulated cell signaling, and improve immune responses. In this review, we evaluate the existing literature and the potential of $\mathrm{N}$-acetylcysteine in promoting cognitive health and alleviating cognitive decline associated with dementia. Discussion will also include possible mechanisms of action of N-acetylcysteine, its effects on aging biology, and safety of long-term use. Based on the available literature, a nutraceutical formulation containing $\mathrm{N}$-acetylcysteine among other compounds has shown some pro-cognitive benefits in Alzheimer's patients and older adults, but the evidence for $\mathrm{N}$-acetylcysteine alone is less robust. Although $\mathrm{N}$-acetylcysteine crosses the blood-brain-barrier, low bioavailability is an obstacle. One promising avenue of research may be to explore derivatives of $\mathrm{N}$-acetylcysteine such as $\mathrm{N}$-acetylcysteine amide, which has been reported in preclinical studies to have higher permeability through cellular and mitochondrial membranes with increased central nervous system bioavailability compared to N-acetylcysteine.
\end{abstract}

Key words: Age-related cognitive decline, NAC, NACA, dementia.

List of abbreviations: COPD: chronic obstructive pulmonary disease; CSF: cerebral spinal fluid; GSH: reduced glutathione; GSSG: oxidized glutathione; MMSE: Mini Mental State Examination; MRS: magnetic resonance spectroscopy; NACA: $N$-acetylcysteine amide.

\section{Introduction}

The brain is an organ that consumes $20 \%$ of the body's energy despite constituting a mere $2 \%$ of one's body weight. High metabolic demands along with low levels of antioxidative defense mechanisms make the brain vulnerable to oxidative damage (1). Indeed, aging and Alzheimer's disease are associated with an imbalance between pro-oxidants and antioxidants, which in turn, can induce oxidative damage to DNA, proteins, and lipids in the brain (2-4). Several studies have highlighted the imbalance in glutathione redox system as a feature of Alzheimer's disease onset and progression. For example, levels of antioxidants including glutathione are significantly lower in mitochondrial and synaptosomal fractions of postmortem brains from Alzheimer's disease patients when compared to age-matched controls (5). In addition, higher levels of oxidative markers (protein carbonyls, 3-nitrotyrosine, 4-hydroxynonenal, and acrolein) significantly correlate with worse cognitive function as measured by the Mini Mental State Examination (MMSE) (5). Brain glutathione concentrations can be measured non-invasively in vivo, using a proton magnetic resonance spectroscopy (MRS). A study using this technology has reported that not only is there a gradual decrease of glutathione levels with aging, but that glutathione levels are significantly depleted in Alzheimer's disease patients (6). Changes in glutathione levels are also apparent peripherally, where people with mild cognitive impairment and Alzheimer's disease have lower glutathione levels and GSH/GSSG ratios in red blood cells (7).

$\mathrm{N}$-acetylcysteine is a precursor of L-cysteine, which in turn is a component of the endogenous antioxidant glutathione, a tripeptide composed of glutamate, cysteine, and glycine. Glutathione (GSH) plays an important role in antioxidant activities, redox (oxidationreduction reaction)-regulated cell signaling, and immune responses (8). The ratio of reduced glutathione (GSH) to oxidized glutathione (GSSG) is often used as a measure of oxidative stress. Because cysteine availability is a limiting factor for GSH synthesis, N-acetylcysteine may help counter oxidative stress by maintaining or 
increasing GSH levels. In addition to being a component of glutathione, the thiol group (S-H) in N-acetylcysteine confers free radical scavenging properties by interacting with reactive oxygen species such as hydroxyl radical $(\bullet \mathrm{OH})$ and hydrogen peroxide $\left(\mathrm{H}_{2} \mathrm{O}_{2}\right)$ (9). $\mathrm{N}$-acetylcysteine is currently used to loosen thick mucus in people with cystic fibrosis or chronic obstructive pulmonary disease (COPD) (10). It is also used as an antidote to acetaminophen and carbon monoxide poisoning (11).

There is a growing number of studies testing the effects of $\mathrm{N}$-acetylcysteine in people with psychiatric or neurological diseases (12). Here, we review the existing literature and evaluate the potential of $\mathrm{N}$-acetylcysteine in promoting cognitive health and preventing dementia.

\section{Main text}

\section{Evaluation of the neuroprotective potential of N-acetylcysteine}

Although no clinical trials have examined whether $\mathrm{N}$-acetylcysteine can prevent cognitive decline or dementia, several clinical trials have evaluated whether it may promote cognitive function. We have subjectively rated the available clinical and preclinical evidence on $\mathrm{N}$-acetylcysteine in Table 1.

Table 1. Subjective rating of clinical and preclinical evidence of $\mathrm{N}$-acetylcysteine for age-related cognitive decline, Alzheimer's disease, and safety of long-term use

\begin{tabular}{|c|c|c|c|c|}
\hline Evidence for: & Types of Evidence & $\begin{array}{l}\text { Potential Benefit }(+) \text { or } \\
\text { Risk (-) }\end{array}$ & Data Quality (A-D) & Summary of Evidence \\
\hline $\begin{array}{l}\text { Protection from age-related } \\
\text { cognitive decline }\end{array}$ & RCT & + & $\mathrm{C}$ & $\begin{array}{l}\text { No studies have looked at prevention } \\
\text { of cognitive decline in humans with } \\
\text { N-acetylcysteine alone, but a nutraceutical } \\
\text { formulation containing N-acetylcysteine has } \\
\text { shown some pro-cognitive benefits in older } \\
\text { adults. }\end{array}$ \\
\hline \multirow[t]{2}{*}{$\begin{array}{l}\text { Protection from } \\
\text { Alzheimer's disease }\end{array}$} & $\mathrm{RCT}$ & + & $\mathrm{C}+$ & $\begin{array}{l}\text { A nutraceutical formulation containing } \\
\text { N-acetylcysteine significantly improved } \\
\text { dementia rating scale and executive function } \\
\text { in Alzheimer's patients. However, treatment } \\
\text { with N-acetylcysteine alone showed less } \\
\text { robust effects, with improvement in letter } \\
\text { fluency task but not the primary outcome } \\
\text { measure (MMSE). }\end{array}$ \\
\hline & Preclinical & + & Not assessed & $\begin{array}{l}\text { Studies in rodent models of Alzheimer's } \\
\text { disease have shown that } \mathrm{N} \text {-acetylcysteine } \\
\text { prevents cognitive impairment. Animal and } \\
\text { in vitro studies have identified plausible } \\
\text { mechanisms of action. }\end{array}$ \\
\hline Safety & $\mathrm{RCT}$ & Excellent & A & $\begin{array}{l}\text { Multiple meta-analyses have reported } \\
\text { that N-acetylcysteine is generally safe and } \\
\text { well-tolerated for most adults. However, } \\
\text { because of its anticoagulant and platelet-in- } \\
\text { hibiting properties, some safety concerns } \\
\text { are noted for patients with increased risk of } \\
\text { bleeding. }\end{array}$ \\
\hline
\end{tabular}

We have subjectively rated the evidence for benefit versus risk of N-acetylcysteine use to prevent or delay age-related cognitive decline and Alzheimer's disease. We independently considered the data from randomized controlled trials (RCTs) and preclinical studies in animal and in vitro models. We also rate the evidence for safety of long-term N-acetylcysteine use. Data quality ranges from high (A) to very low (D) within the context of each type of data. We considered variables of study quality such as sample sizes, consistency of conclusions across studies, duration of treatment and follow-up, and reliable objective measurements of cognitive function as a primary outcome of the study. These ratings are subjective and should be interpreted as a guide rather than a definitive conclusion. The following is a guideline for how data quality was graded.

RCT evidence:

A: Robust replicated results from at least 2 large long-term double-blind RCT, ideally with confirmation from a systematic review or meta-analysis

B: Supporting evidence from the primary outcome of at least one large long-term double-blind RCT

C: A lack of supporting evidence from the primary outcome of a large long-term double-blind RCT but promising results from either small pilot RCTs or from post-hoc and planned secondary analyses.

D: A lack of supporting evidence from quality RCTs 


\section{Human research evaluating the effects of $\mathrm{N}$-acetylcysteine on cognitive functions}

No studies have looked at prevention of cognitive decline or dementia in humans with $\mathrm{N}$-acetylcysteine alone. In a placebo-controlled trial of 34 people with mild cognitive impairment, daily consumption of a nutraceutical formulation containing $600 \mathrm{mg}$ of $\mathrm{N}$-acetylcysteine (along with folate, alpha-tocopherol, vitamin B12, S-adenosyl methionine, and acetyl-Lcarnitine) for 6 months was associated with improvement in dementia rating scale (Cohen's effect size, 0.76) and preservation of executive function (13). The placebo group did not improve in dementia rating scale and declined in executive function, but no direct statistical comparisons were made between the nutraceutical treatment group and the placebo group, making the results less conclusive. In a randomized controlled trial of 93 community-dwelling adults, treatment with the same nutraceutical formulation for 3 months was associated with improved verbal learning compared to the placebo group and improved executive function compared to baseline (14). Patients receiving the placebo showed no improvement. Both the placebo and nutraceutical groups improved further during a 3-month open-label extension of the formulation. Also, performance declined to baseline following withdrawal of the treatment, and statistically improved when participants resumed the formulation. It is currently unknown which of the compounds are responsible for these positive effects, and whether the compounds have additive or synergistic effects.

Some studies have tested the effects of $\mathrm{N}$-acetylcysteine alone, but in people with psychiatric disorders. In a double-blind randomized controlled trial of 46 bipolar disorder patients, $\mathrm{N}$-acetylcysteine $(2000 \mathrm{mg} / \mathrm{d})$ treatment for 6 months did not result in any significant differences in cognitive measures including digit span, word learning, trail making, and verbal fluency when compared with the placebo group (15). There were also no within-group differences between baseline and after $\mathrm{N}$-acetylcysteine treatment. A Cochrane meta-analysis examined randomized controlled trials of various supplements purported to improve cognitive symptoms in people with schizophrenia, of which 2 trials tested the effects of $\mathrm{N}$-acetylcysteine (16). It concluded that the trials were not adequately powered and that evidence for cognitive protection with $\mathrm{N}$-acetylcysteine is limited.

\section{$N$-acetylcysteine research in dementia patients}

In a double-blind randomized controlled trial of 106 Alzheimer's disease patients, 3 or 6 months of the nutraceutical formulation (same as above) resulted in significant improvements in the dementia rating scale and executive function compared to the placebo group (17). During the 6-month open-label extension, both groups improved or maintained cognitive performance.

In contrast, a clinical trial specifically testing $\mathrm{N}$-acetylcysteine in 43 patients with probable Alzheimer's disease reported that $\mathrm{N}$-acetylcysteine $(50 \mathrm{mg} / \mathrm{kg} /$ day) for 6 months failed to significantly alter MMSE scores, which were the primary outcome measures (18). However, the N-acetylcysteine group showed significant benefit on letter fluency task compared to placebo at 6 months and there were trends toward improvement in memory. The treatment effect favored the $\mathrm{N}$-acetylcysteine group for most tasks as well as for a composite measure derived from individual tests. However, the letter fluency task was the only secondary outcome measure that showed statistically significant difference between the treatment and placebo groups.

It is worth noting the controversy that surrounds antioxidant treatments for dementia. A double-blind randomized controlled trial testing an antioxidant combination treatment (vitamin $\mathrm{E}$, vitamin $\mathrm{C}$, and $\alpha$-lipoic acid) in Alzheimer's disease patients reported that this treatment was associated with accelerated cognitive decline as measured by the MMSE, despite a decrease in the level of oxidative stress (F2-isoprostane) in the cerebral spinal fluid (CSF) (19). The study showed no differences between the antioxidant treatment versus placebo groups in CSF biomarkers of Alzheimer's pathology, including $A \beta 42$, tau, and P-tau(181). Thus, cognitive changes with this combination treatment may not be due to worsening of Alzheimer's-related pathology. The mechanisms behind the accelerated cognitive decline with this antioxidant combination are currently unclear. It is also unknown whether $\mathrm{N}$-acetylcysteine may have adverse cognitive effects when combined with other antioxidants.

\section{Mechanisms of action for neuroprotection identified from laboratory and clinical research}

$\mathrm{N}$-acetylcysteine crosses the blood-brain-barrier in both humans and rodents $(20,21)$ and it is a membranepermeable cysteine precursor that does not require active transport via the alanine-serine-cysteine system (8). A single intravenous infusion of $\mathrm{N}$-acetylcysteine (150 mg/ kg) increased blood and brain glutathione concentrations in patients with Parkinson's disease, Gaucher disease, and healthy controls (22). Brain glutathione concentrations were measured using 7T magnetic resonance spectroscopy, and GSH/GSSG redox ratios were measured from blood samples.

Numerous studies in rodents have shown that $\mathrm{N}$-acetylcysteine has pro-cognitive and neuroprotective effects. Chronic administration of $\mathrm{N}$-acetylcysteine (100 $\mathrm{mg} / \mathrm{kg}$, s.c.) improved cognition in a mouse model of accelerated aging (SAMP8 mice) (20). In a mouse model of Alzheimer's disease (streptozotocin-injected), $\mathrm{N}$-acetylcysteine treatment (50 mg/kg/day, p.o.) prevented cognitive impairment and the decrease in 
glucose uptake in the hippocampus (23).

An amide form of $\mathrm{N}$-acetylcysteine ( $\mathrm{N}$-acetylcysteine amide; NACA) is thought to have higher permeability through cellular and mitochondrial membranes, with increased central nervous system bioavailability (24). In a rat model of traumatic brain injury, NACA treatment for 15 days significantly improved cognitive function and cortical tissue sparing when compared to $\mathrm{N}$-acetylcysteine treatment or vehicle (25). NACA also reduced oxidative damage (4-hydroxynonenal levels) at 7 days post-injury and maintained levels of mitochondrial glutathione and mitochondrial bioenergetics. No clinical studies have tested NACA yet, but it may hold greater promise for neuroprotection than $\mathrm{N}$-acetylcysteine.

\section{Effects of N-acetylcysteine on aging biology}

High levels of the amino acid homocysteine are common in people over 65 and have been linked to a wide variety of age-related problems including dementia, stroke, and coronary artery disease (26). In a double-blind randomized controlled trial of 82 people, $\mathrm{N}$-acetylcysteine treatment ( $1.8 \mathrm{~g} /$ day from Fluimucil capsules) for 4 weeks significantly lowered plasma concentrations of homocysteine by $-11.7 \% \pm 3.0 \%$ (placebo: $4.1 \% \pm 3.6 \%$ ) while increasing those of cysteine by $28.1 \% \pm 5.7 \%$ (placebo: $4.0 \% \pm 3.4 \%$ ). N-acetylcysteine also significantly decreased systolic and diastolic blood pressure. It is worth noting that no direct comparisons were made between the $\mathrm{N}$-acetylcysteine group and the placebo group. Also, N-acetylcysteine-treated people had higher baseline homocysteine levels and blood pressure, making the results less conclusive.

Several preclinical studies have evaluated the effects of $\mathrm{N}$-acetylcysteine on lifespan. $\mathrm{N}$-acetylcysteine added to drinking water $(5 \mathrm{~g} / \mathrm{L}$ and $10 \mathrm{~g} / \mathrm{L})$ at 7 months of age increased total and maximum lifespan in male mice, but not in female mice (27). In males and females, both doses caused a sudden drop in body weight followed by continued slower-rate decline in weight such that at 900 days these mice weighed comparably to mice that underwent dietary restriction.

In C. elegans, $\mathrm{N}$-acetylcysteine significantly extended both the mean (by 30.5\%) and maximum lifespan (by 8 days) (28). $\mathrm{N}$-acetylcysteine supplementation also increased the total number of progeny produced. Expression of stress-responsive genes (sod-3 and hsp16.2) increased significantly following $\mathrm{N}$-acetylcysteine. It appears to promote longevity in C. elegans through increased resistance to environmental stressors.

\section{Safety of $\mathrm{N}$-acetylcysteine supplementation}

$\mathrm{N}$-acetylcysteine is generally considered safe and is well-tolerated for most adults. A meta-analysis based on 13 clinical studies including a total of 4,155 bronchitis/COPD patients reported that $\mathrm{N}$-acetylcysteine supplementation for up to 3 years does not increase the risk of side effects (relative risk, $0.94,95 \%$ CI, 0.88-0.99) (29). In a double-blind randomized controlled study of 21 patients with cystic fibrosis, $\mathrm{N}$-acetylcysteine at doses of up to $2800 \mathrm{mg} /$ day was well-tolerated and deemed safe (30). The nutraceutical formulation used in older people and those with mild cognitive impairment was also welltolerated and no serious adverse events were reported for any of the $300+$ participants $(13,17,18)$.

However, some safety concerns have been raised in patients with serious health conditions. In a large metaanalysis of 41 randomized controlled trials including 2,768 patients with systemic inflammatory response syndrome or sepsis, late application (more than 24 hours after the onset of symptoms) of N-acetylcysteine was associated with cardiovascular instability (31). A double-blind randomized controlled trial of 177 patients with renal insufficiency undergoing cardiac surgery reported that $\mathrm{N}$-acetylcysteine treatment $(100 \mathrm{mg} / \mathrm{kg}$ bolus followed by $20 \mathrm{mg} / \mathrm{kg} / \mathrm{hr}$ infusion) was associated with significantly greater blood loss and a requirement of 1.6 more units of blood transfusion $(95 \%$ CI, 0.4-3.1 units) (32). Another double-blind randomized controlled trial of 20 patients undergoing surgery for abdominal aortic aneurysm reported that $\mathrm{N}$-acetylcysteine was associated with anticoagulant and platelet-inhibiting properties, with decreased prothrombin time and prolonged coagulation time (33). These concerns need to be taken into account when $\mathrm{N}$-acetylcysteine treatment is considered for patients with increased bleeding risk.

Some drug interactions with $\mathrm{N}$-acetylcysteine have been observed. N-acetylcysteine should not be administered with nitroglycerin, as the combination can cause severe hypotension (34) and headache (35). N-acetylcysteine interacts with inhaled insulin (Afrezza $^{\mathrm{TM}}$, Exubera $^{\mathrm{TM}}$ ) and may affect the absorption of insulin into the blood stream (36).

\section{Ongoing clinical trials testing $N$-acetylcysteine}

Many clinical trials are ongoing, though none are in Alzheimer's disease or dementia patients. An openlabel trial is testing whether a 3-month $\mathrm{N}$-acetylcysteine supplementation in older adults improves glutathione levels, body composition, mitochondrial energetics, dyslipidemia, oxidative stress, protein/glucose metabolism, cognition, and memory (37). A randomized double-blind clinical trial is testing whether intravenous (once per week) and daily oral $\mathrm{N}$-acetylcysteine treatment supports brain function in patients with Parkinson's disease (38). Another trial is testing whether $\mathrm{N}$-acetylcysteine affects inflammatory and oxidative stress biomarkers in patients with tobacco use disorders and bipolar disorder (39). And a phase III study is testing whether $\mathrm{N}$-acetylcysteine (oral) and/or isotonic bicarbonate (i.v.) prevents serious adverse outcomes following angiographic procedures (40). Finally, another 
clinical trial is testing the role of $\mathrm{N}$-acetylcysteine as an adjuvant to opioid treatment in patients with chronic neuropathic pain (41). A few clinical trials testing the effects of $\mathrm{N}$-acetylcysteine on cognitive functions are planned in Australia as well, one in noncardiac surgical patients (post-anesthesia) (42) and another in schizophrenia patients (43).

\section{Conclusions}

A nutraceutical formulation containing $\mathrm{N}$-acetylcysteine among other compounds has shown some pro-cognitive benefits in Alzheimer's patients and older adults, but the evidence for $\mathrm{N}$-acetylcysteine alone is much weaker. Alzheimer's disease patients have decreased glutathione levels and increased oxidative stress $(7,44)$. N-acetylcysteine is an attractive compound as it is a precursor and component of the endogenous antioxidant glutathione in addition to being a free radical scavenger. While N-acetylcysteine crosses the bloodbrain-barrier, low bioavailability is a concern like other antioxidants. One promising avenue of research may be to explore derivatives of $\mathrm{N}$-acetylcysteine such as NACA (amide form), which has been reported to have higher permeability through cellular and mitochondrial membranes with increased central nervous system bioavailability compared to N-acetylcysteine (24). NACA has shown some promising neuroprotective effects in a rodent model of traumatic brain injury (25) but no studies have tested it in humans yet. In clinical practice, safety is based on low bioavailability of N-acetylcysteine. Safety and dosages will need to be redefined if NACA is used in humans. In order to monitor target engagement and treatment response to $\mathrm{N}$-acetylcysteine or its derivatives, glutathione levels in the brain can be measured using proton magnetic resonance spectroscopy (45). Peripheral levels of GSH and GSH/GSSG ratios can also be measured from blood samples.

Acknowledgements: This work was supported by a generous donation to the Alzheimer's Drug Discovery Foundation by an anonymous foundation.

Conflict of interest: The authors do not declare financial or non-financial competing interests.

\section{References}

1. Jovanovic Z. Antioxidative defense mechanisms in the aging brain. Arch Biol Sci 2017;66, 245-252

2. Mecocci P, MacGarvey U, Beal MF. Oxidative damage to mitochondrial DNA is increased in Alzheimer's disease. Ann Neurol 1994;36, 747-751. https: / / www.ncbi.nlm.nih.gov/pubmed/7979220

3. Montine TJ, Neely MD, Quinn JF et al. Lipid peroxidation in aging brain and Alzheimer's disease. Free Radic Biol Med 2002;33, 620-626.https://www. ncbi.nlm.nih.gov/pubmed/12208348

4. Smith CD, Carney JM, Starke-Reed PE et al. Excess brain protein oxidation and enzyme dysfunction in normal aging and in Alzheimer disease. Proc Natl Acad Sci U S A 1991;88, 10540-10543.https://www.ncbi.nlm.nih.gov/ pubmed / 1683703

5. Ansari MA, Scheff SW. Oxidative stress in the progression of Alzheimer disease in the frontal cortex. J Neuropathol Exp Neurol 2010;69, 155-167. https: / / www.ncbi.nlm.nih.gov/pubmed/20084018

6. Mandal PK, Tripathi M, Sugunan S. Brain oxidative stress: detection and mapping of anti-oxidant marker 'Glutathione' in different brain regions of healthy male/female, MCI and Alzheimer patients using non-invasive magnetic resonance spectroscopy. Biochem Biophys Res Commun 2012;417, 43-48.https: / / www.ncbi.nlm.nih.gov/ pubmed/22120629

7. Bermejo P, Martin-Aragon S, Benedi J et al. Peripheral levels of glutathione and protein oxidation as markers in the development of Alzheimer's disease from Mild Cognitive Impairment. Free Radic Res 2008;42, 162-170.https:// www.ncbi.nlm.nih.gov/pubmed/18297609

8. Bavarsad Shahripour R, Harrigan MR, Alexandrov AV. N-acetylcysteine (NAC) in neurological disorders: mechanisms of action and therapeutic opportunities. Brain Behav 2014;4, 108-122.https://www.ncbi.nlm.nih.gov/ pubmed $/ 24683506$

9. Aruoma OI, Halliwell B, Hoey BM et al. The antioxidant action of $\mathrm{N}$-acetylcysteine: its reaction with hydrogen peroxide, hydroxyl radical, superoxide, and hypochlorous acid. Free Radic Biol Med 1989;6, 593-597. https: / / www.ncbi.nlm.nih.gov/pubmed/2546864

10. Shen Y, Cai W, Lei S et al. Effect of high/low dose N-acetylcysteine on chronic obstructive pulmonary disease: a systematic review and metaanalysis. COPD 2014;11, 351-358.https://www.ncbi.nlm.nih.gov/ pubmed $/ 24378052$

11. Green JL, Heard KJ, Reynolds KM et al. Oral and Intravenous Acetylcysteine for Treatment of Acetaminophen Toxicity: A Systematic Review and Metaanalysis. West J Emerg Med 2013;14, 218-226.https://www.ncbi.nlm.nih. gov/pubmed/23687539

12. Deepmala, Slattery J, Kumar $\mathrm{N}$ et al. Clinical trials of $\mathrm{N}$-acetylcysteine in psychiatry and neurology: A systematic review. Neurosci Biobehav Rev 2015;55, 294-321.https: / / www.ncbi.nlm.nih.gov/pubmed/ 25957927

13. Remington R, Lortie JJ, Hoffmann $\mathrm{H}$ et al. A Nutritional Formulation for Cognitive Performance in Mild Cognitive Impairment: A Placebo-Controlled Trial with an Open-Label Extension. J Alzheimers Dis 2015;48, 591-595. https: / / www.ncbi.nlm.nih.gov/pubmed/26402075

14. Chan A, Remington R, Kotyla E et al. A vitamin/nutriceutical formulation improves memory and cognitive performance in community-dwelling adults without dementia. J Nutr Health Aging 2010;14, 224-230.https:/ / www.ncbi. nlm.nih.gov/pubmed/20191258

15. Dean OM, Bush AI, Copolov DL et al. Effects of $\mathrm{N}$-acetyl cysteine on cognitive function in bipolar disorder. Psychiatry Clin Neurosci 2012;66, 514517.https:/ / www.ncbi.nlm.nih.gov/pubmed/23066769

16. Magalhaes PV, Dean O, Andreazza AC et al. Antioxidant treatments for schizophrenia. Cochrane Database Syst Rev 2016;2, CD008919.https:/ / www. ncbi.nlm.nih.gov/pubmed/26848926

17. Remington R, Bechtel C, Larsen D et al. A Phase II Randomized Clinical Trial of a Nutritional Formulation for Cognition and Mood in Alzheimer's Disease. J Alzheimers Dis 2015;45, 395-405.https://www.ncbi.nlm.nih.gov/ pubmed/25589719

18. Adair JC, Knoefel JE, Morgan N. Controlled trial of N-acetylcysteine for patients with probable Alzheimer's disease. Neurology 2001;57, 1515-1517. https: / / www.ncbi.nlm.nih.gov/ pubmed/11673605

19. Galasko DR, Peskind E, Clark CM et al. Antioxidants for Alzheimer disease: a randomized clinical trial with cerebrospinal fluid biomarker measures. Arch Neurol 2012;69, 836-841.https: / / www.ncbi.nlm.nih.gov/pubmed/22431837

20. Farr SA, Poon HF, Dogrukol-Ak D et al. The antioxidants alpha-lipoic acid and $\mathrm{N}$-acetylcysteine reverse memory impairment and brain oxidative stress in aged SAMP8 mice. J Neurochem 2003;84, 1173-1183.https://www.ncbi. nlm.nih.gov/pubmed/12603840

21. Katz M, Won SJ, Park Y et al. Cerebrospinal fluid concentrations of $\mathrm{N}$-acetylcysteine after oral administration in Parkinson's disease. Parkinsonism Relat Disord 2015;21, 500-503.https:/ / www.ncbi.nlm.nih.gov/ pubmed $/ 25765302$

22. Holmay MJ, Terpstra M, Coles LD et al. N-Acetylcysteine boosts brain and blood glutathione in Gaucher and Parkinson diseases. Clin Neuropharmacol 2013;36, 103-106.https: / / www.ncbi.nlm.nih.gov/pubmed/23860343

23. Costa M, Bernardi J, Fiuza T et al. N-acetylcysteine protects memory decline induced by streptozotocin in mice. Chem Biol Interact 2016;253, 10-17. https: / / www.ncbi.nlm.nih.gov/ pubmed / 27087133

24. Sunitha K, Hemshekhar M, Thushara RM et al. N-Acetylcysteine amide: a derivative to fulfill the promises of N-Acetylcysteine. Free Radic Res 2013;47, 357-367.https: / / www.ncbi.nlm.nih.gov/ pubmed/23472882

25. Pandya JD, Readnower RD, Patel SP et al. N-acetylcysteine amide confers neuroprotection, improves bioenergetics and behavioral outcome following TBI. Exp Neurol 2014;257, 106-113.https://www.ncbi.nlm.nih.gov/ pubmed / 24792639

26. Kuo HK, Sorond FA, Chen JH et al. The role of homocysteine in multisystem age-related problems: a systematic review. The journals of gerontology Series A, Biological sciences and medical sciences 2005;60, 1190-1201.http://www. ncbi.nlm.nih.gov/pubmed/16183962

27. Flurkey K, Astle CM, Harrison DE. Life extension by diet restriction and $\mathrm{N}$-acetyl-L-cysteine in genetically heterogeneous mice. The journals of gerontology Series A, Biological sciences and medical sciences 2010;65, 12751284.https: / / www.ncbi.nlm.nih.gov/ pubmed/20819793

28. Oh SI, Park JK, Park SK. Lifespan extension and increased resistance to environmental stressors by N-acetyl-L-cysteine in Caenorhabditis elegans. Clinics (Sao Paulo) 2015;70, 380-386.https://www.ncbi.nlm.nih.gov/ 
pubmed $/ 26039957$

29. Cazzola M, Calzetta L, Page $\mathrm{C}$ et al. Influence of $\mathrm{N}$-acetylcysteine on chronic bronchitis or COPD exacerbations: a meta-analysis. Eur Respir Rev 2015;24, 451-461.https: / / www.ncbi.nlm.nih.gov/ pubmed / 26324807

30. Dauletbaev N, Fischer P, Aulbach B et al. A phase II study on safety and efficacy of high-dose $\mathrm{N}$-acetylcysteine in patients with cystic fibrosis. Eur J Med Res 2009;14, 352-358.https://www.ncbi.nlm.nih.gov/ pubmed/19666395

31. Szakmany T, Hauser B, Radermacher P. N-acetylcysteine for sepsis and systemic inflammatory response in adults. Cochrane Database Syst Rev, 2012 CD006616.https: / / www.ncbi.nlm.nih.gov/pubmed/22972094

32. Wijeysundera DN, Karkouti K, Rao V et al. N-acetylcysteine is associated with increased blood loss and blood product utilization during cardiac surgery. Crit Care Med 2009;37, 1929-1934.https://www.ncbi.nlm.nih.gov/ pubmed / 19384218

33. Niemi TT, Munsterhjelm E, Poyhia R et al. The effect of N-acetylcysteine on blood coagulation and platelet function in patients undergoing open repair of abdominal aortic aneurysm. Blood Coagul Fibrinolysis 2006;17, 29-34. https:/ / www.ncbi.nlm.nih.gov/pubmed/16607076

34. Horowitz JD, Henry CA, Syrjanen ML et al. Nitroglycerine/N-acetylcysteine in the management of unstable angina pectoris. Eur Heart J 1988;9 Suppl A, 95-100.https:/ / www.ncbi.nlm.nih.gov/pubmed/3137075

35. Ardissino D, Merlini PA, Savonitto S et al. Effect of transdermal nitroglycerin or N-acetylcysteine, or both, in the long-term treatment of unstable angina pectoris. J Am Coll Cardiol 1997;29, 941-947.https:/ / www.ncbi.nlm.nih.gov/ pubmed / 9120179

36. NAC (acetylcysteine) Drug Interactions. Drugscom.https://www.drugs. $\mathrm{com} /$ drug-interactions/acetylcysteine,nac-index.html
37. Sekhar RV. Energetics and Function in Older Humans, 2015. Clinicaltrialsgov. https: / / clinicaltrials.gov/ct2/show/study/NCT02348762

38. Monti DA, Newberg AB, Kremens D et al. Physiological Effects of Nutritional Support in Patients With Parkinson's Diseas, 2015. Clinicaltrialsgov.https:/ / clinicaltrials.gov/ct2/show/study/NCT02445651

39. Bonifacio KL, Rossaneis AC. The Effect of N- Acetylcysteine on Inflammatory and Oxidative Stress Biomarkers (nac), 2015. Clinicaltrialsgov.https:// clinicaltrials.gov/ct2/show/study/NCT02420418

40. Weisbord SD. Prevention of Serious Adverse Events Following Angiography (PRESERVE) 2011. Clinicaltrialsgov.https://clinicaltrials.gov/ct2/show / study / NCT01467466

41. Svikis DS. The Role of N-acetyl-1-cysteine (NAC) as an Adjuvant to Opioid Treatment in Patients With Chronic Neuropathic Pain 2013. Clinicaltrialsgov. https:/ / clinicaltrials.gov/ct2/show/ NCT01840345

42. Skvarc DR, Dean OM, Byrne LK et al. The Post-Anaesthesia N-acetylcysteine Cognitive Evaluation (PANACEA) trial: study protocol for a randomised controlled trial 2016. Trials 17, 395.https://www.ncbi.nlm.nih.gov/ pubmed / 27502769

43. Rossell SL, Francis PS, Galletly C et al. N-acetylcysteine (NAC) in schizophrenia resistant to clozapine: a double blind randomised placebo controlled trial targeting negative symptoms. BMC Psychiatry 2016;16, 320. https: / / www.ncbi.nlm.nih.gov/pubmed / 27629871

44. Huang WJ, Zhang X, Chen WW. Role of oxidative stress in Alzheimer's disease. Biomed Rep 2016;4, 519-522.https://www.ncbi.nlm.nih.gov/ pubmed $/ 27123241$

45. Landheer K, Schulte R, Geraghty B et al. Diffusion-weighted J-resolved spectroscopy, 2016. Magn Reson Med.https://www.ncbi.nlm.nih.gov/ pubmed $/ 27797114$ 